\title{
CLOSED-FORM SOLUTION FOR PIEZOELECTRIC LAYER WITH TWO COLLINEAR CRACKS PARALLEL TO THE BOUNDARIES
}

\author{
B. M. SINGH, J. ROKNE, AND R. S. DHALIWAL \\ Received 6 February 2005; Revised 16 November 2005; Accepted 26 March 2006
}

We consider the problem of determining the stress distribution in an infinitely long piezoelectric layer of finite width, with two collinear cracks of equal length and parallel to the layer boundaries. Within the framework of reigning piezoelectric theory under mode III, the cracked piezoelectric layer subjected to combined electromechanical loading is analyzed. The faces of the layers are subjected to electromechanical loading. The collinear cracks are located at the middle plane of the layer parallel to its face. By the use of Fourier transforms we reduce the problem to solving a set of triple integral equations with cosine kernel and a weight function. The triple integral equations are solved exactly. Closed form analytical expressions for stress intensity factors, electric displacement intensity factors, and shape of crack and energy release rate are derived. As the limiting case, the solution of the problem with one crack in the layer is derived. Some numerical results for the physical quantities are obtained and displayed graphically.

Copyright (c) 2006 B. M. Singh et al. This is an open access article distributed under the Creative Commons Attribution License, which permits unrestricted use, distribution, and reproduction in any medium, provided the original work is properly cited.

\section{Introduction}

Research in the area of piezoelectricity has led to the development of a variety of important electronic and electromechanical devices which are being used in spacecraft launch vehicles and military equipment. When piezoelectric ceramics are subject to mechanical and electrical loads in service, flaws or defects caused by manufacturing may lead to premature failure of these materials (or composite) to static antiplane shear and in plane electric loading which have been studied by several authors. For example, Pak [5] addressed the plane and antiplane fractured problems of an infinite piezoelectric body and obtained the closed form solutions of the stress field and electric displacement near the crack tip. Pak [6] suggested the closed form solution for an infinite piezoelectric body under antiplane loading by employing a complex variable approach. Shindo et al. $[9,10]$ obtained a solution for the infinite strip, parallel or perpendicular to the crack under

Hindawi Publishing Corporation

Mathematical Problems in Engineering

Volume 2006, Article ID 91846, Pages 1-16

DOI 10.1155/MPE/2006/91846 
antiplane loading using the integral transforms. Park and Sun [7] obtained the closed form solutions for all three modes of fracture for an infinite piezoelectric medium containing a center crack subjected to a combined mechanical and electrical loading. Furthermore, Shin et al. [8] and Kwon and Lee [3] considered the case of an eccentric crack in a piezoelectric strip and a central crack in a rectangular piezoelectric body, respectively.

Recently, Li and Duan [4] presented closed form solution for a mode-III crack situated at the mid-plane of a piezoelectric layer. This paper is a further extension of the work discussed in [4].

As we know, an analytic solution in closed-form has some advantages over numerical and approximate solutions, so that in many cases, analytical solutions in closed form are desired for accurate analysis and design. Moreover, analytical solution can serve as a benchmark for the purpose of judging the accuracy and efficiency of various numerical and approximate methods. However owing to the mathematical complexity, certain practical problems of complicated configurations are only solved with the recourse to numerical schemes and it is difficult to obtain their analytical solutions in closed form.

This paper is concerned with the two collinear cracks parallel to the boundaries and embedded symmetrically in a piezoelectric layer under mode III. The top and bottom surfaces of the layer are kept at constant potential difference and displaced by the constant displacement. With the help of Fourier transforms, the solution of the problem is reduced into triple integral equations whose solution is obtained in the closed form. With the aid of a closed form solution, all quantities including the distribution of stress and electric displacement, the crack sliding displacement, the intensity factors for stress and electroelastic field, and energy release rate are determined in closed form. Solution of the problem of a single crack in a layer is also derived in closed form.

\section{Formation of the problem and basic equations}

Consider a rectangular Cartesian coordinate system such that the cross-section of the layer of the strip is $-\infty<x<\infty,-\delta<y<\delta$, and the cracks are located at $a<x<b,-b<$ $x<-a, y=0$. The cracks are infinitely long in the $z$ direction. Because of the assumed symmetry in geometry and loading, it is sufficient to consider the problem for the region $0<x<\infty, 0<y<\delta$, only. The piezoelectric boundary value problem is simplified if we consider only the out-of-plane displacement and the in-plane electric fields in the form

$$
\begin{gathered}
u_{x}=u_{z}=0, \quad u_{y}=w(x, y), \quad E_{x}=E_{x}(x, y), \\
E_{y}=E_{y}(x, y), \quad E_{z}=0,
\end{gathered}
$$

where $u_{i}$ and $E_{i}(i=x, y, z)$ are displacement and electric field vectors, respectively. For this case, there is only nonvanishing the out-of-plane displacement $w(x, y)$ and the inplane electric potential $\phi(x, y)$, which are independent of $z$ and obey the following basic governing differential equations for the antiplane piezoelectricity:

$$
\begin{aligned}
c_{44} \nabla^{2} w+e_{15} \nabla^{2} \phi & =0, \\
e_{15} \nabla^{2} w-\epsilon_{11} \nabla^{2} \phi & =0,
\end{aligned}
$$


in the absence of body force and free charges where $c_{44}, \epsilon_{11}$, and $e_{15}$ are the electric modulus, the dielectric permittivity, and the piezoelectric constant of the piezoelectric layer; and $\nabla^{2}=\partial / \partial x^{2}+\partial / \partial y^{2}$ represents the two-dimensional operator. The components of antiplane shear stress, in-plane electric displacement, and electric field in this layer are given by the following equations:

$$
\begin{array}{rlrl}
\sigma_{z x}=c_{44} \frac{\partial w}{\partial x}+e_{15} \frac{\partial \phi}{\partial x}, & \sigma_{z y} & =c_{44} \frac{\partial w}{\partial y}+e_{15} \frac{\partial \phi}{\partial y} \\
D_{x}=e_{15} \frac{\partial w}{\partial x}-\epsilon_{11} \frac{\partial \phi}{\partial x}, & D_{y}=e_{15} \frac{\partial w}{\partial y}-\epsilon_{11} \frac{\partial \phi}{\partial y} \\
E_{x}=-\frac{\partial \phi}{\partial x}, & E_{y}=-\frac{\partial \phi}{\partial y} .
\end{array}
$$

In this paper we consider a piezoelectric layer subjected to the following electromechanical loading on the boundaries:

$$
w(x, \pm \delta)= \pm w_{0}, \quad \phi(x, \pm \delta)=\mp \phi_{0}, \quad-\infty<x<\infty,
$$

where $w_{0}$ and $\phi_{0}$ are constants. That is, let the top and bottom surfaces of the layer, $y=$ $\pm \delta$, be clamped and displaced along the $z$-direction by an amount $\pm w_{0}$, and there is a constant potential difference between the top and bottom surfaces. Geometry of the problem is shown in Figure 2.1.

In addition to the boundary conditions (2.4), elastic and electric boundary conditions along the crack can be written as

$$
\begin{gathered}
w(x, 0)=0, \quad 0<|x|<a,|x|>b, \\
\sigma_{z y}(x, 0)=0, \quad a<|x|<b, \\
E_{x}(x, 0)=E_{x}^{c_{1}}(x, 0), \quad a<|x|<b, \\
\phi(x, 0)=0, \quad 0<|x|<a, b<|x|,
\end{gathered}
$$

where we assume that the cracks are filled with electrolyte solution and the superscript $c_{1}$ denotes the quantities inside the crack.

In the present study the boundary conditions (2.7) and (2.8) are due to permeable crack.

By making use of Fourier transform it is easy to show that appropriate solution of (2.2) is given by

$$
\begin{aligned}
& w(x, y)=\left(\frac{2}{\pi}\right)^{1 / 2} F_{c}\left[\frac{A(\xi) \sinh [(\delta-y) \xi]}{\sinh (\xi \delta)} ; \xi \longrightarrow x\right]+\frac{w_{0} y}{\delta} \\
& \phi(x, y)=\left(\frac{2}{\pi}\right)^{1 / 2} F_{c}\left[\frac{B(\xi) \sinh [(\delta-y) \xi]}{\sinh (\xi \delta)} ; \xi \longrightarrow x\right]-\frac{\phi_{0} y}{\delta}
\end{aligned}
$$

where $A(\xi)$ and $B(\xi)$ are unknown functions to be determined from the boundary conditions. As usual, $F_{s}$ and $F_{c}$ denote the operators of the Fourier sine and cosine transforms, 


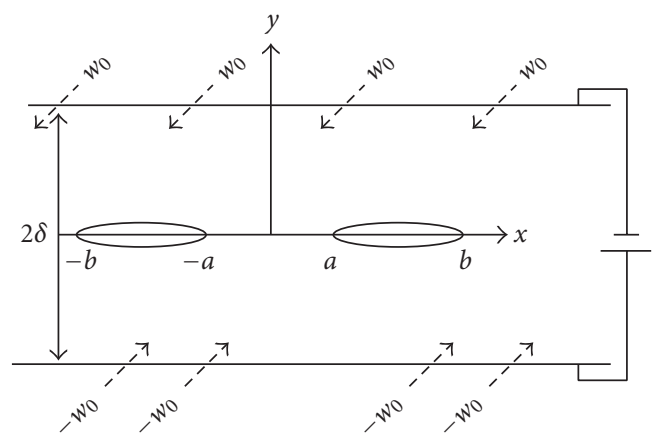

Figure 2.1. Piezoelectric layer with the two collinear cracks subjected to in-plane electric loads and antiplane mechanical loads.

respectively, defined by

$$
\begin{aligned}
& F_{c}\left[\phi_{1}(\xi, y) ; \xi \longrightarrow x\right]=\sqrt{\frac{2}{\pi}} \int_{0}^{\infty} \phi_{1}(\xi, y) \cos (\xi x) d \xi \\
& F_{s}\left[\phi_{1}(\xi, y) ; \xi \longrightarrow x\right]=\sqrt{\frac{2}{\pi}} \int_{0}^{\infty} \phi_{1}(\xi, y) \sin (\xi x) d \xi .
\end{aligned}
$$

By virtue of the constitutive equations (2.9) and (2.10), we find expressions for the antishear stress in plane electric displacement and electric field in the following form:

$$
\begin{gathered}
\sigma_{z y}(x, y)=-\sqrt{\frac{2}{\pi}}\left\{F c\left[\xi\left\{c_{44} A(\xi)+e_{15} B(\xi)\right\} \frac{\cosh [(\delta-y) \xi]}{\sinh (\delta \xi)} ; \xi \rightarrow x\right]\right\}+\frac{c_{44} w_{0}-e_{15} \phi_{0}}{\delta} \\
D_{y}(x, y)=-\sqrt{\frac{2}{\pi}}\left\{F c\left[\xi\left\{e_{15} A(\xi)-\epsilon_{11} B(\xi)\right\} \frac{\cosh [(\delta-y) \xi]}{\sinh (\delta \xi)} ; \xi \rightarrow x\right]\right\}+\frac{e_{15} w_{0}+\epsilon_{11} \phi_{0}}{\delta} \\
E_{x}(x, 0)=-\frac{\partial \phi}{\partial x}=\sqrt{\frac{2}{\pi}} F_{s}\left[\xi \frac{B(\xi) \sinh [(\delta-y) \xi]}{\sinh (\delta \xi)} ; \xi \rightarrow x\right] \\
E_{y}(x, 0)=-\frac{\partial \phi}{\partial y}=\sqrt{\frac{2}{\pi}} F_{c}\left[\xi \frac{B(\xi) \cosh [(\delta-y) \xi]}{\sinh (\delta \xi)} ; \xi \longrightarrow x\right]+\frac{\phi_{0}}{\delta}
\end{gathered}
$$

Substituting (2.9), (2.10), (2.12), (2.14) into the boundary conditions (2.5)-(2.8), we find $B(\xi)=0$ and the following triple integral equations:

$$
\begin{gathered}
F_{c}[A(\xi) ; \xi \longrightarrow x]=0, \quad x \in I_{1}, I_{2}, \\
F_{c}[\xi A(\xi) \operatorname{coth}(\delta \xi) ; \xi \longrightarrow x]=\sqrt{\frac{\pi}{2}} P, \quad x \in I_{2},
\end{gathered}
$$


B. M. Singh et al. 5

where

$$
\begin{gathered}
P=\frac{c_{0}}{\delta c_{44}}, \quad c_{0}=c_{44} w_{0}-e_{15} \phi_{0}, \\
I_{1}=\{x \mid 0<x<a\}, \quad I_{2}=\{x \mid a<x<b\}, \quad I_{3}=\{x \mid b<x<\infty\} .
\end{gathered}
$$

Following Dhaliwal et al. [1, page 750], the solution of the triple integral equations (2.16) can be written as

$$
A(\xi)=\xi^{-1} \int_{a}^{b} \phi(t) \sin (t \xi) d t
$$

where

$$
\begin{gathered}
\phi(t)=\frac{1}{\sqrt{[\cosh (2 c b)-\cosh (2 c t)][\operatorname{coth}(2 c t)-\operatorname{coth}(2 c a)]}} \\
\times\left[P(\cosh (2 c t)-\cosh (2 c a))+2 c^{2} C_{1}\right], \\
C_{1}=\frac{P \sinh ^{2}(c a)}{c^{2} F(\pi / 2, r)}\left[F\left(\frac{\pi}{2}, r\right)-\Pi\left(\frac{\pi}{2}, r_{1}, r\right)\right], \quad c a>0, \\
c=\frac{\pi}{2 \delta}, \quad r=\frac{\left(\sinh ^{2}(c b)-\sinh ^{2}(c a)\right)^{1 / 2}}{\sinh ^{1 / 2}(c) \cosh ^{2}(c a)}, \\
r_{1}=\left[\frac{\sinh ^{2}(c b)-\sinh ^{2}(c a)}{\sinh ^{2}(c b)}\right],
\end{gathered}
$$

$F(\cdot)$ is the elliptic integral of first kind defined as

$$
F\left(\phi_{1}, k\right)=\int_{0}^{\phi_{1}} \frac{d \alpha}{\sqrt{1-k^{2} \sin ^{2} \alpha}},
$$

and $\Pi(\cdot)$ is the elliptic integral of the third kind defined as

$$
\Pi\left(\phi_{1}, n, k\right)=\int_{0}^{\phi_{1}} \frac{d \alpha}{\left(1-n \sin ^{2} \alpha\right) \sqrt{1-k^{2} \sin ^{2} \alpha}} .
$$

Following [1] we can easily find that

$$
C_{1} \longrightarrow 0 \quad \text { as } a \longrightarrow 0 \text {. }
$$

Also making use of [1] the alternative form of the solution of the triple integral equations (2.16) may be written in the following form:

$$
\begin{aligned}
\phi(t)= & \frac{1}{[(\cosh (2 c t)-\cosh (2 c a))(\cosh (2 c b)-\cosh (2 c t))]^{1 / 2}} \\
& \times\left[-P(\cosh (2 c b)-\cosh (2 c t))+2 c^{2} C_{2}\right],
\end{aligned}
$$


6 Closed-form solution for cracked piezoelectric layer

where

$$
C_{2}=\frac{P}{c^{2} F(\pi / 2, r)}\left[\sinh ^{2}(c b) F\left(\frac{\pi}{2}, r\right)-\sinh ^{2}(c a) \Pi\left(\frac{\pi}{2}, r_{1}, r\right)\right], \quad c a>0 .
$$

Substituting the values of $C_{1}$ and $C_{2}$ into (2.20) and (2.26), we find that

$$
\phi(t)=\frac{P\left[\sinh ^{2}(c t)-\sinh ^{2}(c a) \Pi\left(\pi / 2, r_{1}, r\right) / F(\pi / 2, r)\right]}{\left[\left(\sinh ^{2}(c t)-\sinh ^{2}(c a)\right)\left(\sinh ^{2}(c b)-\sinh ^{2}(c t)\right)\right]^{1 / 2}}, \quad a<t<b, c a>0 .
$$

\section{Shear stress, electric displacements, stress intensity factors, shape of crack}

We can write (2.12) formally as

$$
\sigma_{z y}(x, y)=\frac{c_{0}}{\delta}-\frac{2}{\pi} c_{44} \frac{\partial}{\partial x} \int_{0}^{\infty} A(\xi) \operatorname{coth}(\delta \xi) \sin (x \xi) d \xi, \quad 0<x<\infty .
$$

Substituting the value of $A(\xi)$ from (2.19) into (3.1) and interchanging the order of integration and using the following integral:

$$
\int_{0}^{\infty} \frac{\operatorname{coth}(\delta \xi) \sin (x \xi) \sin (t \xi) d \xi}{\xi}=\frac{1}{2} \log \left|\frac{\tanh (c t)+\tanh (c x)}{\tanh (c t)-\tanh (c x)}\right|
$$

which can be easily obtained from the book of Gradshteyn and Ryzhik [2, page 516], we find that

$$
\begin{aligned}
{\left[\sigma_{z y}(x, 0)\right]_{0<x<a} } & =\frac{c_{0}}{\delta}-\frac{c_{44}}{\delta} \int_{a}^{b} \frac{\sinh (2 c t) \phi(t) d t}{\cosh (2 c t)-\cosh (2 c x)}, \\
{\left[\sigma_{z y}(x, 0)\right]_{x>b} } & =\frac{c_{0}}{\delta}+\frac{c_{44}}{\delta} \int_{a}^{b} \frac{\sinh (2 c t) \phi(t) d t}{\cosh (2 c x)-\cosh (2 c t)} .
\end{aligned}
$$

Substituting the value of $\phi(t)$ from (2.26) and (2.20) into (3.3), we find that

$$
\begin{gathered}
{\left[\sigma_{z y}(x, 0)\right]_{0<x<a}=\frac{c_{44}\left[P(\cosh (2 c b)-\cosh (2 c x))-2 c^{2} C_{2}\right]}{\sqrt{[\cosh (2 c b)-\cosh (2 c x)][\operatorname{coth}(2 c a)-\operatorname{coth}(2 c x)]}},} \\
{\left[\sigma_{z y}(x, 0)\right]_{x>b}=\frac{c_{44}\left[P(\cosh (2 c x)-\cosh (2 c a))+2 c^{2} C_{1}\right]}{\sqrt{[\cosh (2 c x)-\cosh (2 c a)][\operatorname{coth}(2 c x)-\operatorname{coth}(2 c b)]}},}
\end{gathered}
$$


B. M. Singh et al. 7

where we have used the following integral:

$$
\begin{aligned}
& \int_{a}^{b} \frac{\sinh (2 c t)[(\cosh (2 c t)-\cosh (2 c a))(\cosh (2 c b)-\cosh (2 c t))]^{-1 / 2}}{\cosh (2 c t)-\cosh (2 c x)} d t \\
& \quad= \begin{cases}\delta[(\cosh (2 c a)-\cosh (2 c x))(\cosh (2 c b)-\cosh (2 c x))]^{-1 / 2}, & 0<x<a, \\
0, & a<x<b, \\
-\delta[(\cosh (2 c x)-\cosh (2 c a))(\cosh (2 c x)-\cosh (2 c b))]^{-1 / 2}, & x>b .\end{cases}
\end{aligned}
$$

Substituting the values of $C_{1}$ and $C_{2}$ from (2.21) and (2.27) into (3.4) and (3.5), we find that

$$
\begin{aligned}
{\left[\sigma_{z y}(x, 0)\right]_{0<x<a}=} & \frac{P c_{44}}{\sqrt{\left[\sinh ^{2}(c b)-\sinh ^{2}(c x)\right]\left[\sinh ^{2}(c a)-\sinh ^{2}(c x)\right]}} \\
& \times\left[\sinh ^{2}(c a) \frac{\Pi}{F}-\sinh ^{2}(c x)\right], \\
{\left[\sigma_{z y}(x, 0)\right]_{x>b}=} & \frac{P c_{44}}{\sqrt{\left[\sinh ^{2}(c x)-\sinh ^{2}(c a)\right]\left[\sinh ^{2}(c x)-\sinh ^{2}(c b)\right]}} \\
& \times\left[\sinh ^{2}(c x)-\frac{\Pi}{F} \sinh ^{2}(c a)\right],
\end{aligned}
$$

where

$$
\begin{aligned}
& F=F\left(\frac{\pi}{2}, r\right), \\
& \Pi=\Pi\left(\frac{\pi}{2}, r_{1}, r\right) .
\end{aligned}
$$

The stress intensity factors at the inner and outer crack tips are defined as

$$
\begin{aligned}
& K_{a}^{\tau}=\lim x \longrightarrow a^{-}\left[\sqrt{2 \pi(a-x)}\left\{\sigma_{y z}(x, 0)\right\}_{0<x<a}\right], \\
& K_{b}^{\tau}=\lim x \longrightarrow b^{+}\left[\sqrt{2 \pi(x-b)}\left\{\sigma_{y z}(x, 0)\right\}_{x>b}\right] .
\end{aligned}
$$

Making use of (3.7) we can write (3.9) in the following form:

$$
\begin{aligned}
& K_{a}^{\tau}=P c_{44} \sqrt{\frac{2 \pi}{c \sinh (2 a c)\left[\sinh ^{2}(c b)-\sinh ^{2}(c a)\right]}} \times\left[\left(\frac{\Pi}{F}-1\right) \sinh ^{2}(c a)\right], \\
& K_{b}^{\tau}=P c_{44} \sqrt{\frac{2 \pi}{c \sinh (2 b c)\left[\sinh ^{2}(c b)-\sinh ^{2}(c a)\right]}} \times\left[\sinh ^{2}(c b)-\frac{\Pi}{F} \sinh ^{2}(c a)\right] .
\end{aligned}
$$


8 Closed-form solution for cracked piezoelectric layer

Making use of (2.13), (2.19), (2.20), (2.21), (2.26), and (2.27) we can easily obtain

$$
\begin{aligned}
{\left[D_{y}(x, 0)\right]_{0<x<a}=} & \frac{1}{\delta}\left\{e_{15} w_{0}+\epsilon_{11} \phi_{0}-\frac{e_{15} c_{0}}{c_{44}}\right\} \\
& +\frac{P e_{15}}{\sqrt{\left[\sinh ^{2}(c b)-\sinh ^{2}(c x)\right]\left[\sinh ^{2}(c a)-\sinh ^{2}(c x)\right]}} \\
& \times\left[\sinh ^{2}(c a) \frac{\Pi}{F}-\sinh ^{2}(c x)\right], \\
{\left[D_{y}(x, 0)\right]_{b<x}=} & \frac{1}{\delta}\left(e_{15} w_{0}+\epsilon_{11} \phi_{0}-\frac{e_{15} c_{0}}{c_{44}}\right) \\
& +\frac{P e_{15}}{\sqrt{\left[\sinh ^{2}(c x)-\sinh ^{2}(c a)\right]\left[\sinh ^{2}(c x)-\sinh ^{2}(c b)\right]}} \\
& \times\left[\sinh ^{2}(c x)-\frac{\Pi}{F} \sinh ^{2}(c a)\right]
\end{aligned}
$$

by using a method similar to the one used in obtaining (3.7).

The intensity factor for electric displacement can be defined as

$$
\begin{aligned}
& K_{a}^{D}=\lim _{x \rightarrow a^{-}}\left[\sqrt{2 \pi(a-x)}\left\{D_{y}(x, 0)\right\}_{0<x<a}\right], \\
& K_{b}^{D}=\lim _{x \rightarrow b^{+}}\left[\sqrt{2 \pi(a-x)}\left\{D_{y}(x, 0)\right\}_{b<x}\right] .
\end{aligned}
$$

Making use of (3.11) we find from (3.12) that

$$
\begin{aligned}
& K_{a}^{D}=P e_{15} \sqrt{\frac{2 \pi}{c \sinh (2 a c)\left[\sinh ^{2}(c b)-\sinh ^{2}(c a)\right]}} \times\left[\left(\frac{\Pi}{F}-1\right) \sinh ^{2}(c a)\right], \\
& K_{a}^{D}=P e_{15} \sqrt{\frac{2 \pi}{c \sinh (2 b c)\left[\sinh ^{2}(c b)-\sinh ^{2}(c a)\right]}} \times\left[\sinh ^{2}(c b)-\frac{\Pi}{F} \sinh ^{2}(c a)\right] .
\end{aligned}
$$

We find from (2.9) that

$$
[w(x, 0)]_{a<x<b}=\left(\frac{2}{\pi}\right) \int_{0}^{\infty} A(\xi) \cos (x \xi) d \xi .
$$

Substituting the value of $A(\xi)$ from (2.19) into (3.14) and interchanging the order of integration, we find that

$$
[w(x, 0)]_{a<x<b}=\int_{x}^{b} \phi(t) d t .
$$


B. M. Singh et al. 9

Substituting for $\phi(t)$ from (2.28) into (3.15), we find that

$$
[w(x, 0)]_{a<x<b}=P \int_{x}^{b} \frac{\left(\sinh ^{2}(c t)-\sinh ^{2}(c a)(\Pi / F)\right) d t}{\sqrt{\left[\sinh ^{2}(c t)-\sinh ^{2}(c a)\right]\left[\sinh ^{2}(c b)-\sinh ^{2}(c t)\right]}} .
$$

Evaluating the above integral with the help of Gradshteyn and Ryzhik [2, pages 242, 266], we obtain that

$$
\begin{gathered}
{[w(x, 0)]_{a<x<b}=-\frac{P}{c \sinh (c b) \cosh (c a)}\left[\frac{\sinh ^{2}(c a) \Pi\left(r_{3}, r\right)}{F}+\cosh ^{2}(c b) \Pi\left(r_{3}, r_{2}, r\right)+F\left(r_{3}, r\right)\right],} \\
r_{2}=\left[\frac{\sinh ^{2}(c a)-\sinh ^{2}(c b)}{\cosh ^{2}(c a)}\right], \\
r_{3}=\sin ^{-1}\left[\frac{\cosh ^{2}(c a)\left[\sinh ^{2}(c b)-\sinh ^{2}(c x)\right]}{\cosh ^{2}(c x)\left[\sinh ^{2}(c b)-\sinh ^{2}(c a)\right]}\right]^{1 / 2} .
\end{gathered}
$$

The antiplane strain is defined as

$$
\gamma_{y z}(x, 0)=\frac{\partial w}{\partial y}=-\frac{2}{\pi} \int_{0}^{\infty} \xi A(\xi) \cot (x \xi) \cos (x \xi) d \xi .
$$

$K_{a}^{\gamma}$ and $K_{b}^{\gamma}$ are the strain intensity factors, and we can easily find that

$$
\begin{aligned}
& K_{a}^{\gamma}=\lim _{x \rightarrow a^{-}}\left[\sqrt{2 \pi(a-x)} \gamma_{y z}\right]=\frac{K_{a}^{\tau}}{c_{44}}, \\
& K_{b}^{\gamma}=\lim _{x \rightarrow b^{+}}\left[\sqrt{2 \pi(x-b)} \gamma_{y z}\right]=\frac{K_{b}^{\tau}}{c_{44}} .
\end{aligned}
$$

Evaluating the energy release rate $G$ for the antiplane case as obtained by Park and Sun [7], we find that

$$
\begin{aligned}
& G_{a}=\frac{K_{a}^{\gamma} K_{a}^{\tau}}{2}=\frac{\left[K_{a}^{\tau}\right]^{2}}{2 c_{44}}, \\
& G_{b}=\frac{K_{b}^{\gamma} K_{b}^{\tau}}{2}=\frac{\left[K_{b}^{\tau}\right]^{2}}{2 c_{44}} .
\end{aligned}
$$

\section{Single crack in an infinite layer}

In what follows we consider a single crack lying in the mid-plane of a piezoelectric layer subjected to boundary conditions (2.4). The corresponding electroelastic field may be derived straightforwardly from the results of Section 3. If we have a single crack of length 
$2 b$ located on the line $y=0,-b<x<b$, of the infinite strip of breath $2 \delta(-\infty<x<$ $\infty,-\delta<y<\delta)$, we let $a \rightarrow 0$, then making use of (2.21) and (2.25), we find that

$$
\phi(t)=\frac{c_{0}}{\delta c_{44}} \frac{\sinh (c t)}{\sqrt{\sinh ^{2}(c b)-\sinh ^{2}(c t)}} .
$$

Now making use of (2.17), (3.5), (3.15), and (4.1), we find that

$$
\begin{aligned}
{\left[\sigma_{y z}(x, 0)\right]_{b<x} } & =\frac{c_{0}}{\delta} \frac{\sinh (c x)}{\sqrt{\sinh ^{2}(c x)-\sinh ^{2}(c b)}}, \\
{[w(x, 0)]_{0<x<b} } & =\frac{2}{\pi} \frac{c_{0}}{\delta} \cos ^{-1}\left[\frac{\sinh (c x)}{\sinh (c b)}\right] \\
K_{b}^{\tau} & =c_{0} \sqrt{\frac{2 \tanh (c b)}{\delta}} .
\end{aligned}
$$

We can easily find with the help of Section 3 the expressions for the electric displacement and intensity factor of electric displacement for $a \rightarrow 0$ in the following form:

$$
\begin{gathered}
{\left[D_{y}(x, 0)\right]_{b<x}=\frac{1}{\delta}\left\{e_{15} w_{0}+\epsilon_{11} \phi_{0}-\frac{e_{15} c_{0}}{c_{44}}\right\}+\frac{c_{0}}{\delta c_{44}} \frac{\sinh (c x)}{\sqrt{\sinh ^{2}(c x)-\sinh ^{2}(c b)}},} \\
K_{b}^{D}=\frac{e_{15} c_{0}}{\delta c_{44}} \sqrt{\frac{2 \tanh (c b)}{\delta}}
\end{gathered}
$$

The energy release rate in this case is

$$
G_{b}^{\tau}=\frac{c_{0}^{2}}{c_{44} \delta} \tanh \left(\frac{\pi b}{2 \delta}\right)
$$

Equations (4.2)-(4.4) are not possible for $\delta \rightarrow \infty$.

The energy release rate for an infinite piezoelectric ceramic can be written in the form

$$
G_{\infty}=\frac{\pi b c_{0}^{2}}{2 c_{44}}
$$

as per Zhang and Tong [11].

\section{Numerical results, discussions, and conclusions}

The numerical values of the stress intensity factors $K_{a}^{\tau}, K_{b}^{\tau}$, the energy release rates $G_{a}, G_{b}$, and the shear stress $\sigma_{y z}(x, 0)$ have been calculated from (3.10), (3.20), (3.7). The numerical values of the material constants taken for these computations are given in Table 5.1.

The numerical values of $K_{a}^{\tau} / c_{0} \sqrt{\pi a}$ and $K_{b}^{\tau} / c_{0} \sqrt{\pi b}$ have been displayed in Figures 5.1, 5.2 , respectively, against $\delta / b$ ( $2 \delta$ is the breadth of the layer) for various values of $a / b=$ $0.1,0.2,0.5,0.7,0.9$. We observe that both the stress intensity factors decrease with the 
B. M. Singh et al. 11

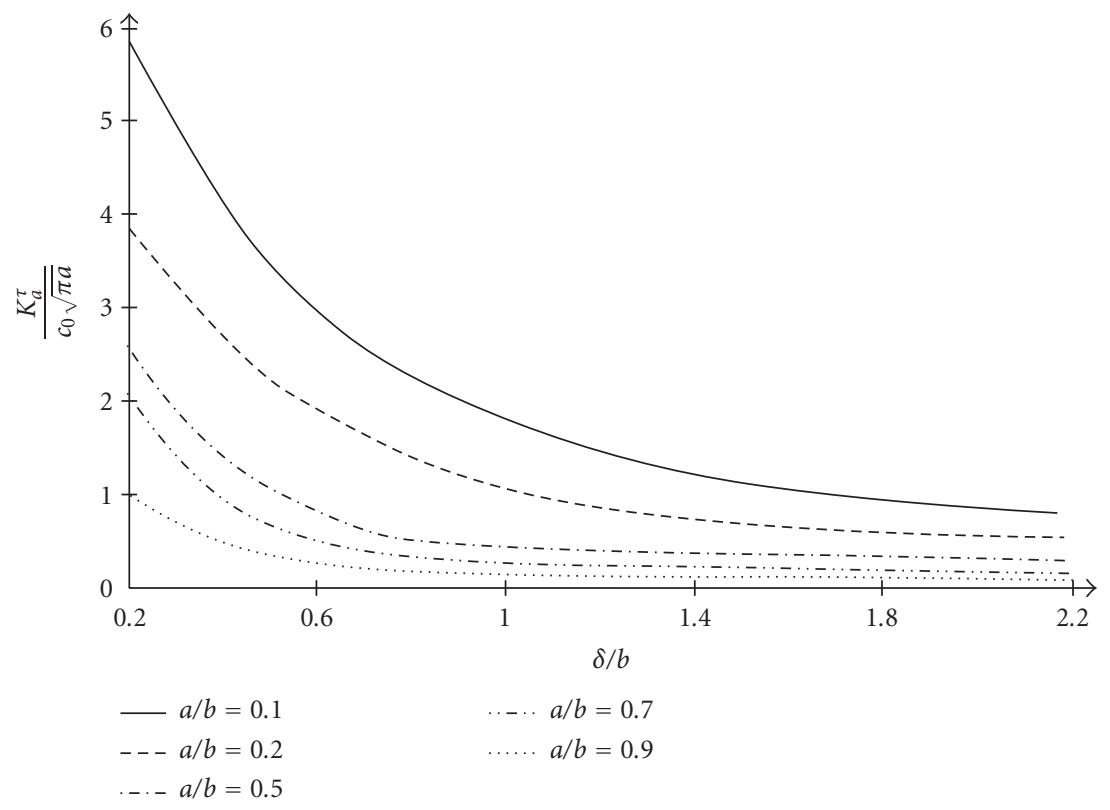

Figure 5.1. Variation of normalized stress intensity factors $K_{a}^{\tau} / c o \sqrt{\pi a}$ with $\delta / b$ for different values of $a / b$.

Table 5.1. The relevant material constants.

\begin{tabular}{lcc}
\hline & PZT-5 & Epoxy \\
\hline$c_{44}\left(\times 10^{10} \mathrm{~N} / \mathrm{m}^{2}\right)$ & 2.11 & 0.18 \\
$e_{15}\left(\mathrm{C} / \mathrm{m}^{2}\right)$ & 12.3 & 0 \\
\hline
\end{tabular}

increase of the layer breadth, and also the stress intensity factors decrease with decreasing the size of crack.

The numerical values of $G_{a} / G_{\infty}$ and $G_{b} / G_{\infty}$ have been displayed in Figures 5.3, 5.4, respectively, against $\delta / b$ for various values of $a / b=0.1,0.2,0.5,0.7,0.9$. We notice that the energy release rates increase with the increase of the layer breadth and they decrease with the decrease of the crack size.

The numerical values of shear stress $\sigma_{y z}(x, 0) / 10$ have been displayed in Figures 5.5, 5.6 against $\delta / b$ for various values of $x / b=0.2,0.3,0.4,1.2,2.0,5.0$. The shear stress decreases as layer breadth increases, and also from Figure 5.6, we find that the shear stress decreases as the point $x$ moves away from either of the crack tips. The numerical values for Figures 5.5, 5.6 have been computed for the material PZT- 5 and $\phi_{0}=w_{0}=1$.

The numerical values of the shear stress $\sigma_{y z}(x, 0) \cdot 10^{-9}$ for the piezoelectric material epoxy have been displayed in Figures 5.7, 5.8. The trend in these graphs is similar to the graphs in Figures 5.5, 5.6. 
12 Closed-form solution for cracked piezoelectric layer

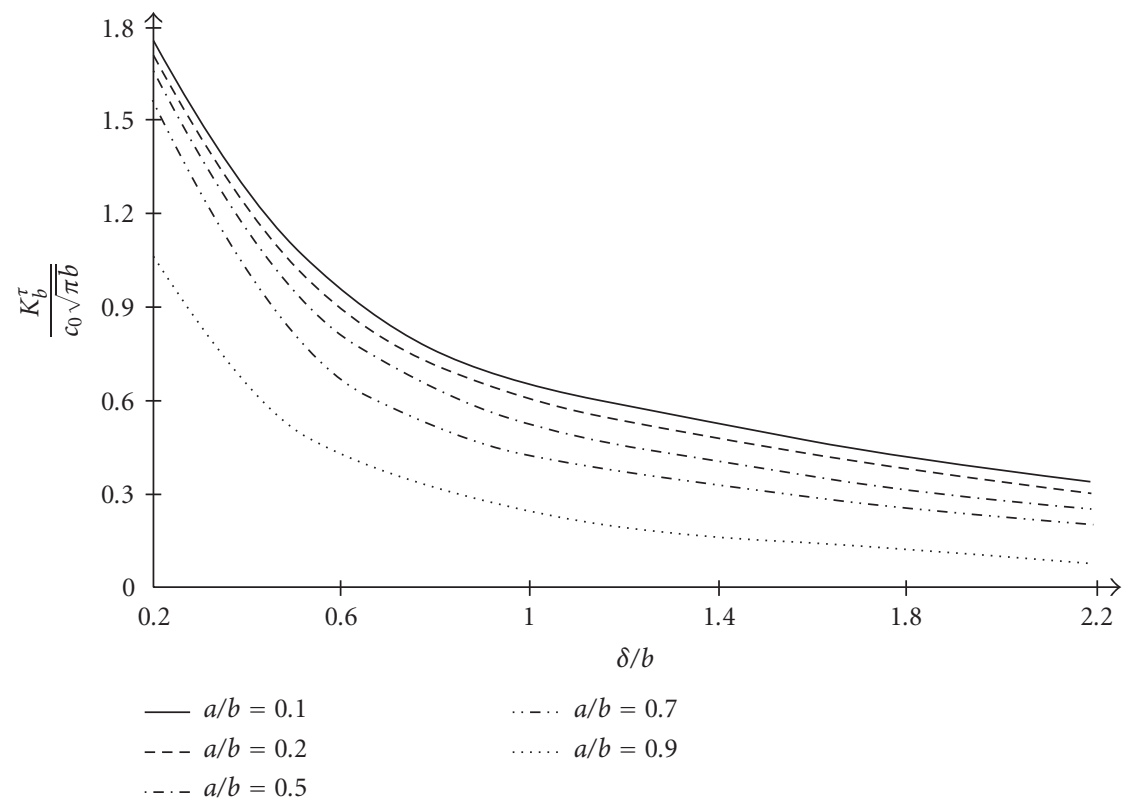

Figure 5.2. Variation of normalized stress intensity factors $K_{b}^{\tau} / c o \sqrt{\pi b}$ with $\delta / b$ for different values of $a / b$.

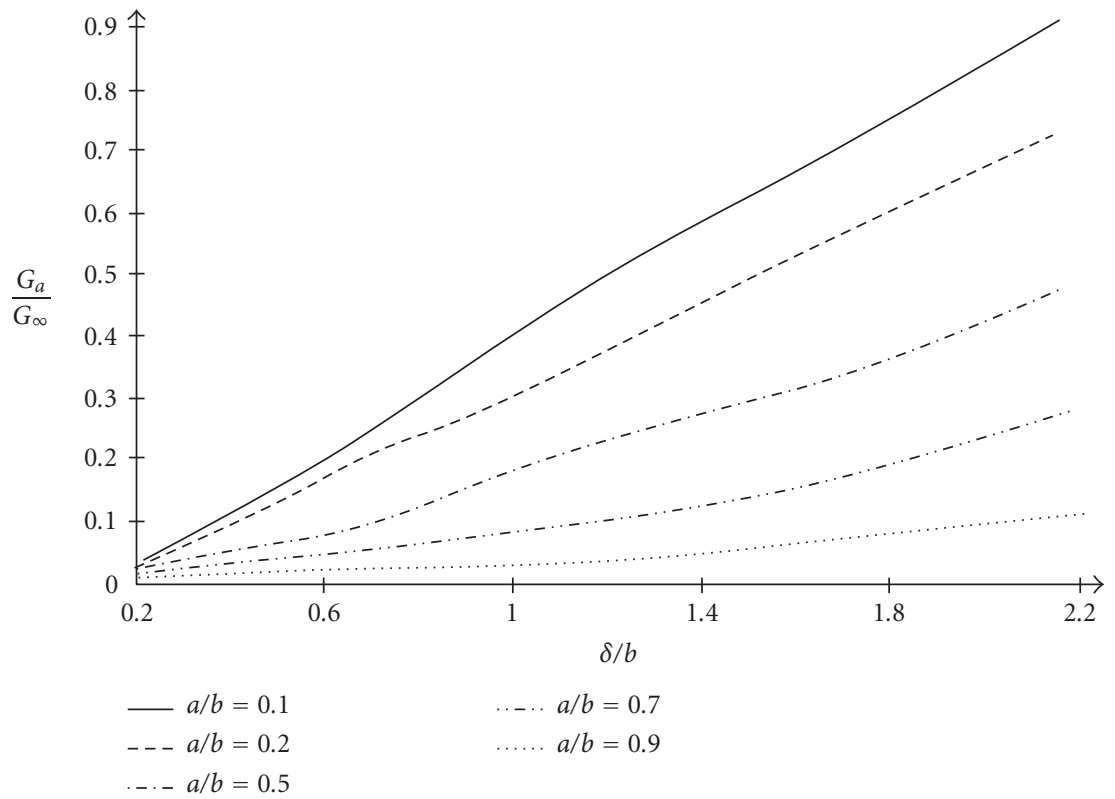

Figure 5.3. Variation of normalized energy release rate $G_{a} / G_{\infty}$ with $\delta / b$ for different values of $a / b$. 
B. M. Singh et al. 13

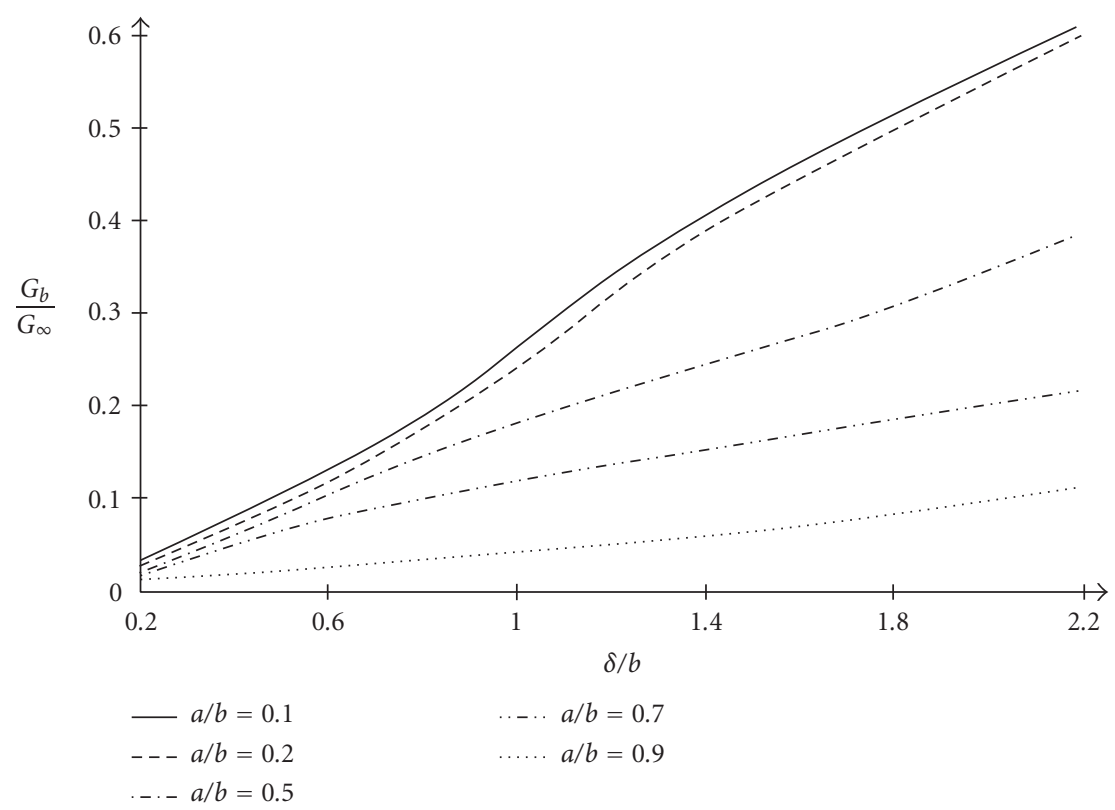

Figure 5.4. Variation of normalized energy release rate $G_{b} / G_{\infty}$ with $\delta / b$ for different values of $a / b$.

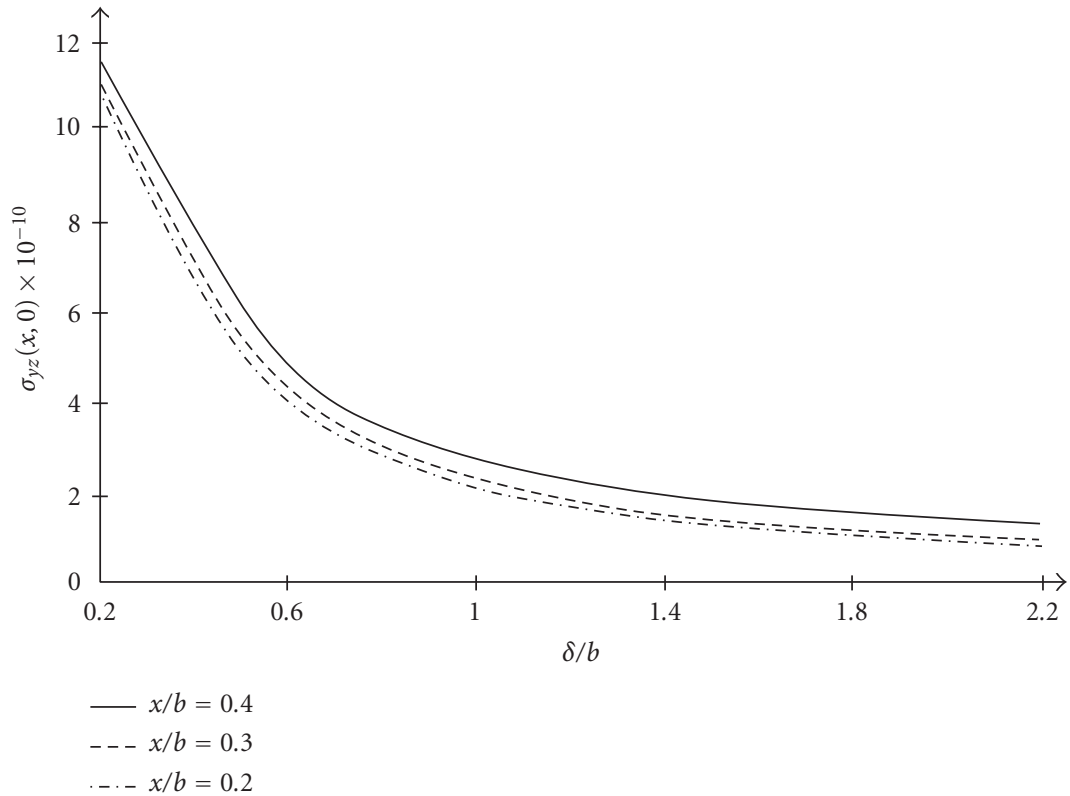

Figure 5.5. Variation of shear stress $\sigma_{y z}(x, 0) \times 10^{-10}$ for piezoelectric material PZT-5 with $\delta / b$ for $x / b=0.4,0.3,0.2, \phi_{0}=w_{0}=1, a=0.5$. 
14 Closed-form solution for cracked piezoelectric layer

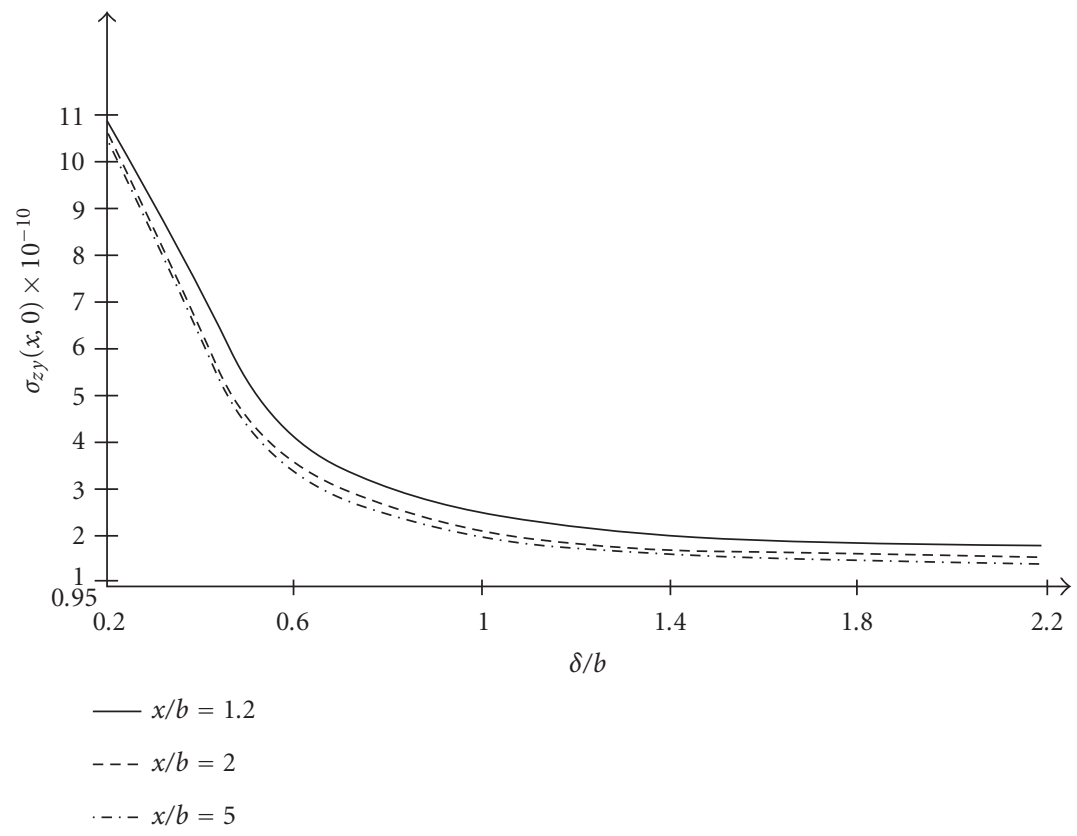

Figure 5.6. Variation of shear stress $\sigma_{y z}(x, 0) \times 10^{-10}$ for piezoelectric material PZT-5 with $\delta / b$ for $x / b=1.2,2.0,5.0, \phi_{0}=w_{0}=1, a=0.5$.

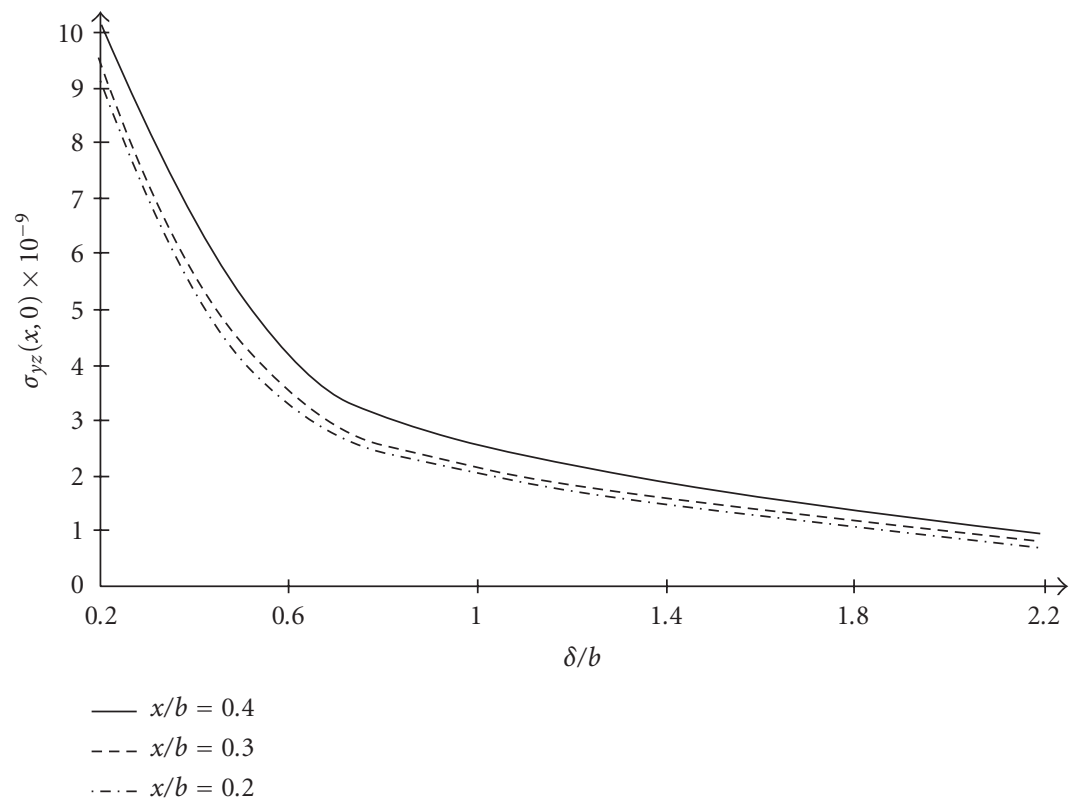

Figure 5.7. Variation of shear stress $\sigma_{y z}(x, 0) \times 10^{-9}$ for piezoelectric material epoxy with $\delta / b$ for $x / b=$ $0.2,0.3,0.4, \phi_{0}=w_{0}=1, a=0.5$. 


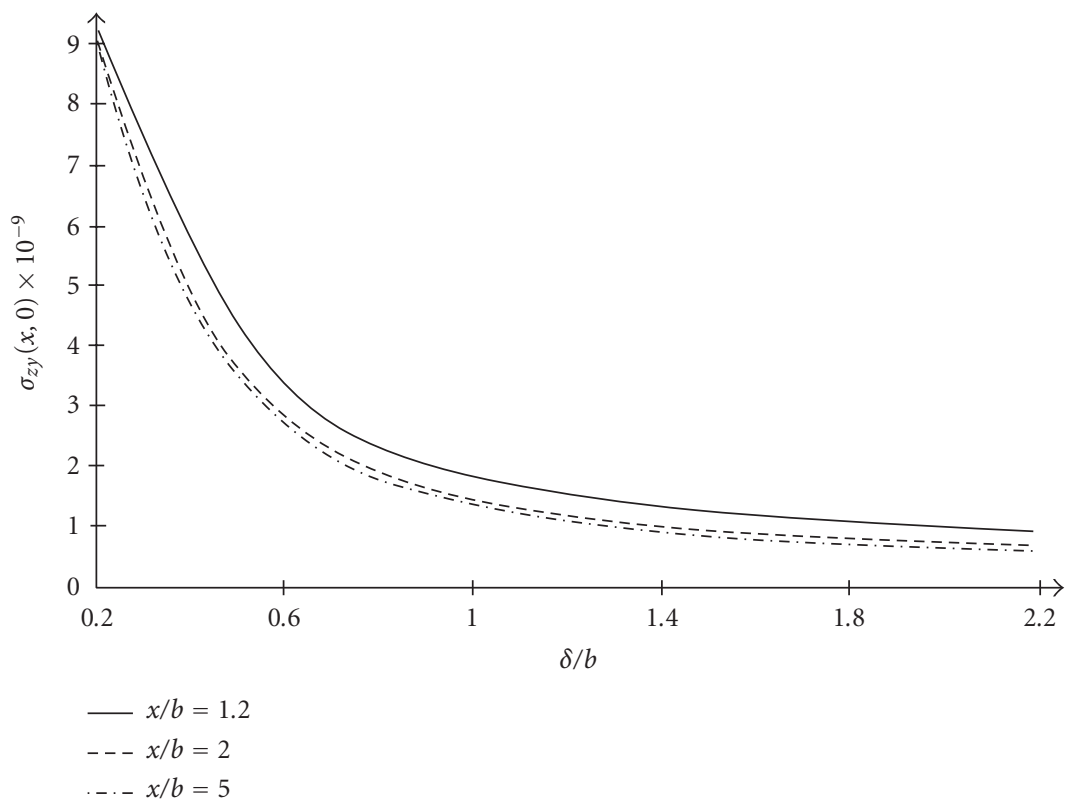

Figure 5.8. Variation of shear stress $\sigma_{y z}(x, 0) \times 10^{-9}$ for piezoelectric material epoxy with $\delta / b$ for $x / b=$ $1.2,2.0,5.0, \phi_{0}=w_{0}=1, a=0.5$.

\section{References}

[1] R. S. Dhaliwal, B. M. Singh, and D. S. Chehil, Two coplanar Griffith cracks under shear loading in an infinitely long elastic layer, Engineering Fracture Mechanics 23 (1986), no. 4, 695-704.

[2] I. S. Gradshteyn and I. M. Ryzhik, Table of Integrals, Series, and Products, Academic Press, New York, 1980.

[3] S. M. Kwon and K. Y. Lee, Analysis of stress and electric fields in a rectangular piezoelectric body with a center crack under anti-plane shear loading, International Journal of Solids and Structures 37 (2000), no. 35, 4859-4869.

[4] X.-F. Li and X.-Y. Duan, Closed-form solution for a mode-III crack at the mid-plane of a piezoelectric layer, Mechanics Research Communications 28 (2001), no. 6, 703-710.

[5] Y. E. Pak, Crack extension force in a piezoelectric materials, Journal of Applied Mechanics 57 (1990), no. 3, 647-653.

[6] __ Linear electro-elastic fracture mechanics of piezoelectric materials, International Journal of Fracture 54 (1992), 79-100.

[7] S. B. Park and C. T. Sun, Effect of electric field on fracture of piezoelectric ceramics, International Journal of Fracture 70 (1995), no. 3, 203-216.

[8] J. W. Shin, S. M. Kwon, and K. Y. Lee, Eccentric crack in a piezoelectric strip under electromechanical loading, Journal of Applied Mechanics 67 (2000), no. 4, 846-847.

[9] Y. Shindo, F. Narita, and K. Tanaka, Electroelastic intensification near anti-plane shear crack in orthotropic piezoelectric ceramic strip, Theoretical and Applied Fracture Mechanics 25 (1996), no. $1,65-71$.

[10] Y. Shindo, K. Tanaka, and F. Narita, Singular stress and electric fields of a piezoelectric ceramic strip with a finite crack under longitudinal shear, Acta Mechanica 120 (1997), no. 1-4, 31-45. 


\section{Closed-form solution for cracked piezoelectric layer}

[11] P.-Y. Zhang and P. Tong, Fracture mechanics for a mode III crack in a piezoelectric material, International Journal of Solids and Structures 33 (1996), no. 3, 343-359.

B. M. Singh: Department of Computer Science, The University of Calgary, Calgary, Alberta, Canada T2N-1N4

J. Rokne: Department of Computer Science, The University of Calgary, Calgary, Alberta, Canada T2N-1N4

E-mail address: rokne@cpsc.ucalgary.ca

R. S. Dhaliwal: Department of Computer Science, The University of Calgary, Calgary, Alberta, Canada T2N-1N4

Current address: Department of Mathematics and Statistics, The University of Calgary, Calgary, Alberta, Canada T2N-1N4

E-mail address: dhali.r@shaw.ca 


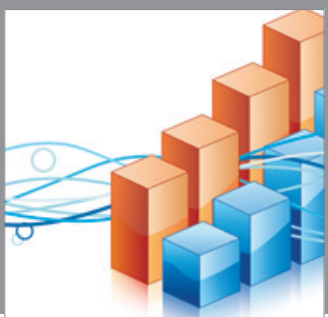

Advances in

Operations Research

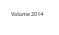

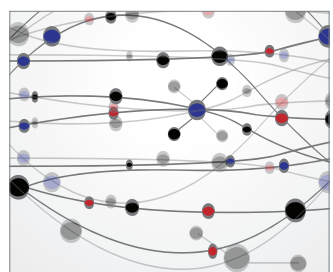

\section{The Scientific} World Journal
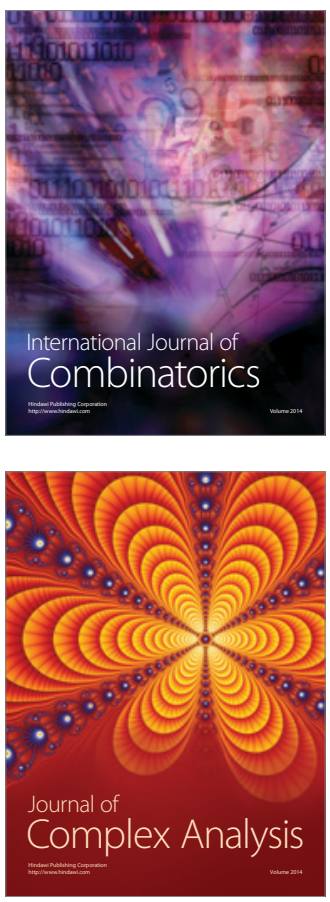

International Journal of

Mathematics and

Mathematical

Sciences
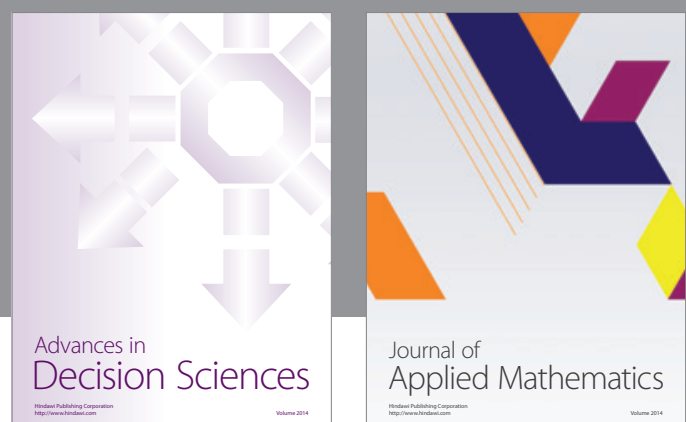

Journal of

Applied Mathematics
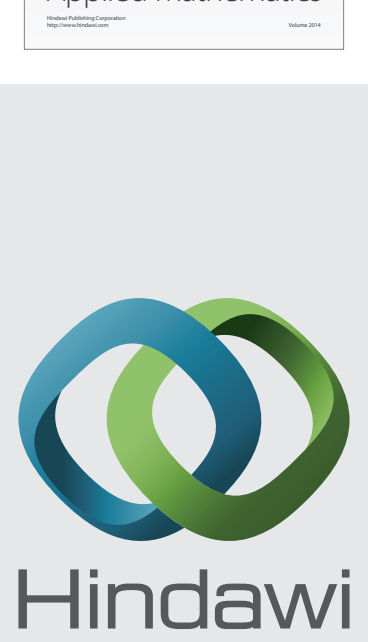

Submit your manuscripts at http://www.hindawi.com
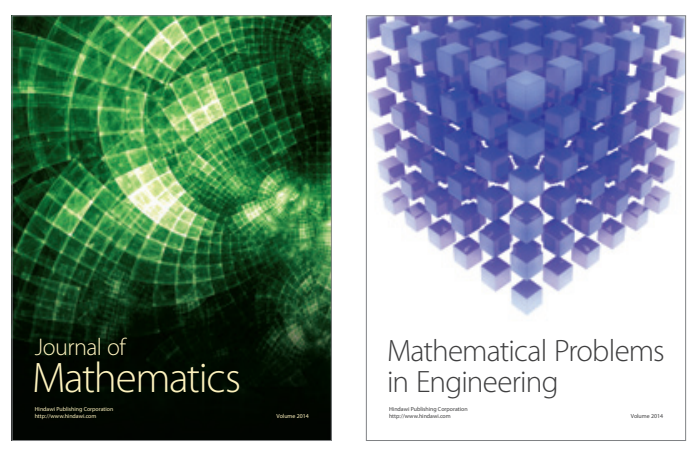

Mathematical Problems in Engineering
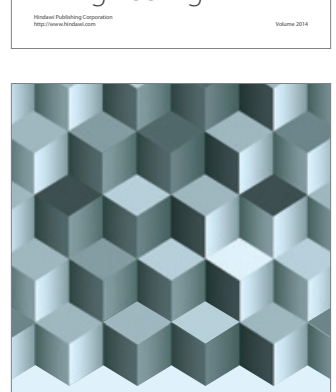

Journal of

Function Spaces
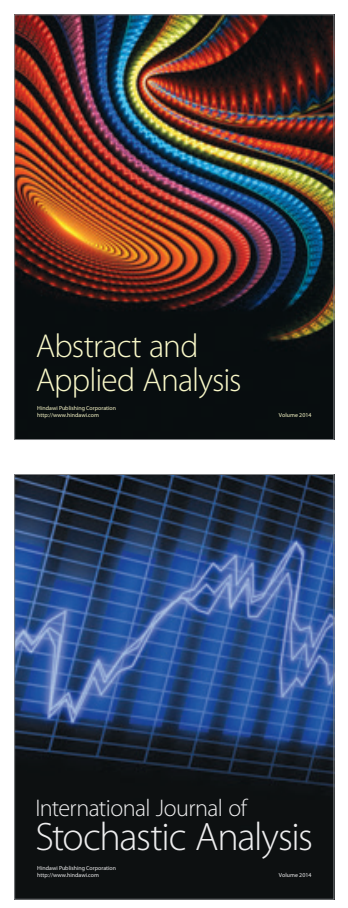

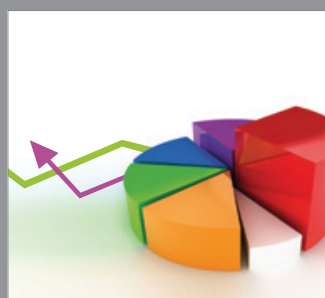

ournal of

Probability and Statistics

Promensencen
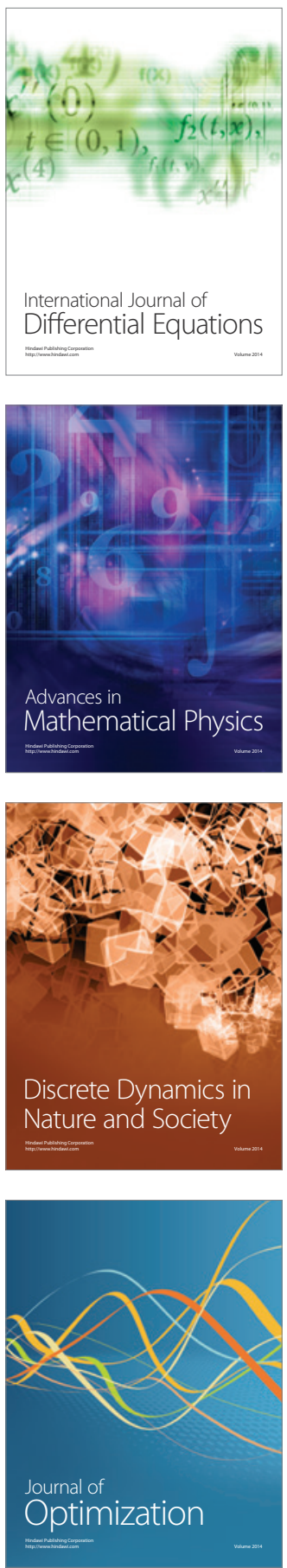